\title{
Warranty analysis of a two-component system with type I stochastic dependence
}

\author{
Nan Zhang ${ }^{\mathrm{a}}$, Mitra Fouladirad ${ }^{\mathrm{a}, *}$, Anne Barros $^{\mathrm{b}}$ \\ ${ }^{a}$ ICD-LM2S, Université de Technologie de Troyes, Troyes, France \\ ${ }^{b}$ Norwegian University of Science and Technology, Trondheim, Norway
}

\begin{abstract}
This paper develops the warranty cost analysis of a two-component in series system with stochastic dependence. From the perspectives of both the manufacturer and the consumer, the short-run total profits and costs under the non-renewing free replacement (non-renewing FRW) policy and the renewing free replacement (RFRW) policy are derived respectively. Numerical examples are given showing the impact of the failure dependence to both the consumer and the manufacturer.
\end{abstract}

Keywords: two-component systems,stochastic dependence,free replacement, the finite time horizon

\section{Introduction}

A warranty is a contractual agreement from the manufacturer to its consumers which requires the manufacturer to compensate its consumers if the sold product fails when properly used within a specified time period which is referred to as warranty period $[4,19,20]$. The compensation may have different forms. For example, the manufacturer takes full charge or shares with the consumer the replacement/repair cost of the product. It is a doubleedged sword to the manufacture which, on the one hand, it is a promotional tool attracting more potential customers by sending the product message to the market regarding its reliability and quality, on the other hand, it is a negative profit to the manufacturer by covering the entire or part of the

\footnotetext{
*Corresponding author

Email address: mitra.fouladirad@utt.fr (Mitra Fouladirad )
} 
product maintenance cost. Therefore, warranty has a significant influence on both the product manufacturer and the consumer. Different perspectives have been investigated in the literature. For instance, the estimation of the arrival rate of the warranty claims, the product lifetime analysis, the warranty cost analysis, the sales lag and reporting analysis, the prediction of warranty claims, etc. More details can be found in [14, 21]. In this study, we examine the cost associated with two frequently-used warranty policies.

In the literature, numerous warranty policies have been considered in the warranty cost analysis modeling. For example, the free replacement/repair policy and the pro rata policy $([27,6,31,10])$. The former means that the product maintenance/replacement costs within the warranty period are covered by the manufacture while the latter indicates that before the warranty expiration the product repair costs depend on the product age at failure. Two types of free replacement/repair policy have been widely considered in the literature: the non-renewing free replacement/repair warranty $([13,25])$ under which the warranty length is fixed and the renewing free replacement/repair warranty $([25,33,5,8,7,12,32])$ which implies that if the product fails within the initial warranty period it is repaired and simultaneously a full warranty is offered. [1] proposed a 'full-service warranty' policy under which the functional component(s) or subsystem(s) were opportunistically repaired at no charge to the consumer within the warranty period. The aforementioned authors examined the manufacture's warranty cost under different system configurations. [24] examined the warranty of multi-component systems regrading the fixed warranty period and imperfect repair.

In the past few years, more attention has been paid to multi-component systems in the the warranty cost analysis. Comparing to the single-component system, the multi-component system is more intractable and intricate. Firstly, the dependencies between components complicate the warranty modeling and the optimization of the warranty policy. Secondly, the opportunistic maintenance can be applied to the system which may be more economic.

It is worth mentioning that in the previously reviewed studies, the analysis of the warranty cost usually focus on the single-component system or the multi-component system with the assumption of possessing independent items. The models regarding the multi-component are always maintenancecost-oriented or system reliability/availability-oriented without mentioning the maintenance cost allocation between for example the product supplier and its consumer. To address this limitations, in this study, we consider the warranty cost allocation between the manufacturer and the consumer where 
the product is modeled by a two component in series system with stochastic dependence between each other.

For the maintenance policy study of multi-component systems regarding stochastic dependence we can list essential papers such as [22] considering 2 types of failure interactions of two-component systems, [23] dealing with a shock damage model known also as the model with the type 3 failure interaction. Since then, numerous maintenance policies and related optimization/system reliability problems of the multi-component system with failure interactions are investigated $([2,11,15,30,34,18])$. For instance, [3] considered the maintenance optimization problem of a two-component system where the system monitoring was imperfect. [16] investigated the condition based maintenance policy of a multi-component system where the failure dependence was modeled by Lévy copulas. It was shown that the stochastic dependence had significant influence on the optimization of the maintenance policy. [28] developed a dynamic opportunistic condition-based maintenance policy for multi-component systems where the degradation level of each component had impact on the remaining useful life of other components. [17] developed the warranty cost analysis of multi-component systems with failure interaction between components under the renewing free replacement policy. The manufacturer's expected warranty costs with different system configurations were examined. Therefore, this paper is motivated by the idea of, from both the perspectives of the manufacturer and the consumer, investigating the warranty costs of a system having inner dependent components.

The main contributions of the paper are

- To propose warranty policy of a two-component system with failure interaction,

- To consider minimal repairs as well as replacements of the whole system,

- To consider the deterioration level of one component and failure rate of the other component

- To propose exact cost calculation based on the renewal theory;

- For the first time the expressions of warranty costs considering failure dependence from two point of views are derived with exact mathematical formulations; 
- The proposed expressions make it more efficient in the estimating of the warranty costs for the manufacture comparing to the simulation-based method;

- The methodology is general and could be applied to the warranty cost analysis when system replacement are involved;

The rest of the paper is organized as follows. Model assumptions, maintenance policies and warranty policies are introduced in section 2. In section 3 , under both the non-renewing free replacement policy and the renewing free replacement policy warranty policy (we will call them non-renewing FRW and RFRW for short hereafter), the warranty costs within the warranty cycle are derived from the perspective of the manufacturer. By assuming that the product service time is finite and fixed, the warranty profits to the manufacturer and the costs to the consumer are obtained. A numerical example with sensitivity analysis is illustrated in section 4. Finally we make our conclusions in section 5 .

\section{Model descriptions and assumptions}

\subsection{General assumptions}

Here we consider a two-component in series system with failure dependence as follows.

- Component 1 is repairable with lifetime distribution $F(t), F(0)=0$, density function $f(t)$ and failure rate $h(t)=\frac{f(t)}{1-F(t)}$.

- Whenever component 1 failure occurs, it induces the failure of component 2 with probability $\bar{r}, 0<\bar{r}<1$ and has no effect on component 2 with probability $r, r+\bar{r}=1$.

- Component 2 is non-repairable and it fails when its deterioration level exceeds a predetermined threshold $L$. The failure of component 2 causes the failure of component 1 .

- Denote $\{Y(t), t \geq 0\}$ be the natural deterioration level of component 2 at time $t$ and $\sigma_{L}$ be the time at which the deterioration level reaches or first exceeds $L, L>0$. Then its distribution function is

$$
G_{\sigma_{L}}(t)=\mathbb{P}\left(\sigma_{L} \leq t\right)=\mathbb{P}(Y(t) \geq L), t \geq 0 .
$$




\subsection{Mathematical formulation of failure probabilities}

For simplification, in the following we call the system failure as

1. minor failure if only component 1 fails;

2. major failure if both component 1 and 2 fail.

Let $N(t)$ be the number of component 1 failures by time $t$ and $p_{n}(t)=$ $\mathbb{P}\{N(t)=n\}$. Then it follows a non-homogeneous Poisson process with

$$
p_{n}(t)=\frac{H(t)^{n}}{n !} e^{-H(t)}
$$

where $H(t)=\int_{0}^{t} h(\theta) \mathrm{d} \theta$.

Let $T_{1}$ be the major failure time with lifetime distribution $F_{s}(\cdot)$. Denoted $\bar{F}_{s}(t)=1-F_{s}(t)$. Then we have

$$
\bar{F}_{s}(t)=\sum_{k=0}^{\infty} r^{k} p_{k}(t) \bar{G}_{\sigma_{L}}(t)
$$

where $\bar{G}_{\sigma_{L}}(t)=1-G_{\sigma_{L}}(t)$. Therefore the major failure time distribution is

$$
F_{s}(t)=1-\sum_{k=0}^{\infty} r^{k} p_{k}(t) \bar{G}_{\sigma_{L}}(t)
$$

\subsection{Maintenance and Warranty assumptions}

Suppose that component 1 is minimally repaired when it fails itself without evoking the failure of component 2. The system is renewed when both component 1 and 2 fail. The component 1 repair cost and the system renewal cost for the manufacturer are $c_{1}$ and $c_{2}$ respectively. The two warranty policies non-renewing FRW and RFRW are described as follows.

Assume that the initial warranty period offered by the manufacturer under both the non-renewing FRW and the RFRW are $W$, during $W$

- under the non-renewing FRW

- component 1 is minimally repaired at its failure time $T_{f 1}$ and the warranty of the system remains valid during the remaining $W-T_{f 1}$ period. 
- the system is replaced when both component 1 and component 2 fail at time $T_{f 2}$ and the warranty of the system remains valid only during the remaining $W-T_{f 2}$ period.

- under the RFRW

- component 1 is minimally repaired at its failure time $T_{f 1}$ and the warranty of the system remains valid during the remaining $W-T_{f 1}$ period.

- the system is replaced when both component 1 and component 2 fail at time $T_{f 2}$ and a full system warranty period of length $W$ is offered.

In the following section, firstly, under both the non-renewing FRW and RFRW policy, the expected warranty costs within the warranty period from the viewpoint of the manufacturer are examined.

\section{Warranty cost analysis}

3.1. The warranty costs of the manufacturer under the non-renewing FRW and $R F R W$ respectively

Assume that the initial warranty period is $W$, the maintenance cost of component 1 and the replacement cost of the system for the manufacturer are $c_{1}$ and $c_{2}$ respectively.

\subsubsection{The warranty costs of the manufacturer under non-renewing FRW}

Theorem 3.1. Under the non-renewing FRW, the expected manufacturer cost $\mathbb{E}(C(W))$ within the warranty period $W$ is given by

$$
\mathbb{E}(C(W))=\int_{0}^{W}[1+M(W-t)] k(t) \mathrm{d} t
$$

where $F_{s}(t)$ is the system renewal time distribution given in equation $2, M(t)$ is the renewal function related with $F_{s}(t)$ denoted by $M(t)=\sum_{n=1}^{\infty} F_{s}^{(n)}(t)$, $k(t)=c_{1} r h(t) \bar{F}_{s}(t)+c_{2} f_{s}(t), f_{s}(t)$ is the intensity function of $F_{s}(t)$.

See Appendix A for the proof. 


\subsubsection{The expected manufacturer's cost under the RFRW policy}

Under the RFRW, the manufacture takes full charge of the warranty cost for the repair/replacement of the failed product during the warranty period and a full warranty is simultaneously offered to the consumer at the system renewal time. In this situation, the total warranty cycle defined as the time period from the initial product purchase time to the end of warranty contract depending on the initial warranty period $W$ and also on the system replacement frequency. Denoted by

- $T_{\text {total }}$ the total warranty cycle,

- $N_{w}$ the system renewal number when the initial warranty period is $W$,

- $\theta_{i}, i=1,2, \cdots, N_{w}$ the inter-arrival major failure times between the $(i-1)$ th system replacement and the $i$ th system renewal.

It can be easily noticed that $\theta_{i}, i=1,2, \cdots, N_{w}$ are identically and independently distributed with distribution function given as follows.

Lemma 1. Let $\theta$ be an inter-arrival major failure time and $W$ the warranty period

$$
\begin{aligned}
F_{\theta}(t) & =\mathbb{P}\left(\theta_{i} \leq t\right)=\mathbb{P}\left(T_{1} \leq t \mid T_{1} \leq W\right) \\
& = \begin{cases}\frac{F_{s}(t)}{F_{s}(W)} & t<W \\
1 & t \geq W\end{cases}
\end{aligned}
$$

where $T_{1}$ is the first system renewal time. Hence the total warranty length can be expressed as

$$
T_{\text {total }}=\sum_{i=1}^{N_{w}} \theta_{i}+W
$$

For example, Figure 1 depicts a possible warranty cycle where $N_{w}=3$ and $T_{\text {total }}=\theta_{1}+\theta_{2}+\theta_{3}+W$. Therefore, the expected warranty cost $\mathbb{E}\left(C^{R}(W)\right)$ for the manufacturer under the RFRW is derived. 


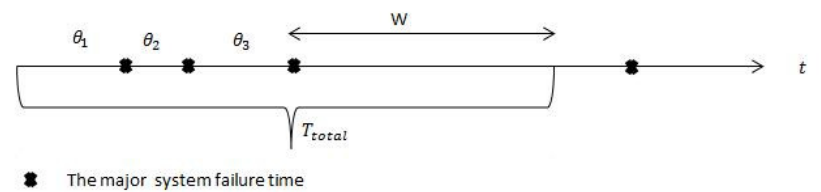

Figure 1: A possible system warranty period under RFRW policy

Theorem 3.2. Under the RFRW, from the viewpoint of the manufacturer, the expected warranty cost within the warranty cycle is

$$
\begin{aligned}
\mathbb{E}\left(C^{R}(W)\right)= & \sum_{n=0}^{\infty} \bar{F}_{s}(W) F_{s}^{n}(W) \\
& \left(n c_{2}+c_{1} r H(W)+n r c_{1} \frac{\int_{0}^{W} H(t) \mathrm{d} F_{s}(t)}{F_{s}(W)}\right)
\end{aligned}
$$

where $F_{s}(t)$ is the system renewal time distribution given in equation $2, h(t)$ and $H(t)$ are the component 1 failure rate and cumulative hazard function respectively.

See Appendix B for the proof.

\subsection{The expected total cost of consumers and total profit of manufacturers on a finite time horizon}

In the previous section, we have focused on the expected warranty cost of the manufacturer within the warranty period. One step further, if we consider the consumer behaviour, suppose that he/she intends to put the product in service for a finite time period $T, W<T<\infty$. Besides, after the warranty period, the consumer prefers coming back to the original manufacturer (or the retailer, or the seller etc. here we held the same regard between them) for the maintenance. Denoted $c_{11}, c_{22}$ be the charges for component 1 repair and system replacement respectively to the consumer. It is reasonable to assume that $c_{i i}>c_{i}, i=1,2$ as the manufacturer makes profits from the disparities between the prices $c_{11}, c_{22}$ and the costs $c_{1}, c_{2}$. In the following, the warranty cost allocation to the consumer and the manufacturer are examined. Assume that the expected warranty cost to the consumer under the RFRW within the finite time horizon $T$ is $\mathbb{E}(C(T))$ and the corresponding expected manufacturer profit is $\mathbb{E}(P(T))$. 
3.2.1. Expected warranty expense analysis under the RFRW

Theorem 3.3. Under the RFRW, the expected maintenance cost of the ownership and the expected total profit of the manufacturer in $[0, T]$ are as follows:

$$
\begin{gathered}
\mathbb{E}(C(T))=\int_{W}^{T}(1+M(T-u)) k(u) \mathrm{d} u \\
\mathbb{E}(P(T))=\mathbb{E}(C(T))-\int_{0}^{T}(1+M(T-t)) k_{1}(t) \mathrm{d} t-c_{2} M(T)
\end{gathered}
$$

where $F_{s}(t)$ is the system renewal time distribution given in equation (2), $k(t)=c_{22} f_{s}(t)+c_{11} \bar{F}_{s}(t) r h(t)$ for $t \geq W$ and 0 otherwise; $k_{1}(t)=c_{1} r \bar{F}_{s}(t) h(t)$, $t>0$ and $M(t)$ is the renewal function related with $F_{s}(t)$ given as $M(t)=$ $\sum_{n=1}^{\infty} F_{s}^{(n)}(t), f_{s}(t)$ is the derivative function of $F_{s}(t)$.

See Appendix C for the proof.

\subsubsection{Expected maintenance cost analysis under the non-renewing FRW}

Under the non-renewing FRW, the product warranty is $W$ and not supposed to be renewed. System failure occurs during $(W, T]$ are taken charge by the consumer. It is reasonable to assume that the consumer is provided by a new product and also a full warranty when he/she replaces the failed system with a new one himself/herself and pays the whole price $c_{22}$ as the initial purchase price including a new product and a full warranty. Denoted by $E_{n o}(C(T))$ and $E_{n o}(P(T))$ the expected warranty cost to the consumer and the expected cost to the manufacturer under the non-renewing FRW when the product service cycle is $T$, we have the following theorem.

Theorem 3.4. Under the non-renewing FRW, the expected warranty cost of the consumer in $[0, T]$ is derived as

$$
\mathbb{E}\left(C_{n o}(T)\right)=K_{0}(T)+\int_{0}^{T} K_{0}(T-u) \mathrm{d} M_{U}(u)
$$

where $K_{0}(t)=c_{22} F_{U}(t)+c_{11} r \int_{W}^{t} \bar{F}_{U}(\theta) h(\theta) \mathrm{d} \theta$ for $t \geq W$ and 0 otherwise; $F_{U}(t)=F_{s}(t)-\int_{0}^{W} \bar{F}(t-x) \mathrm{d} M(x)$ for $t \geq W$ and 0 otherwise; and $M_{U}(t)$ is the renewal function related with $F_{U}(t)$.

If $F \in I F R$, the manufacturer profit $E_{\text {no }}(P(T))$ satisfies

$$
\mathbb{E}\left(P_{\text {no }}(T)\right) \geq \mathbb{E}_{\text {low }}(P(T))
$$


where $\mathbb{E}_{\text {low }}(P(T))=\mathbb{E}\left(C_{n o}(T)\right)-c_{1} r H(T)-c_{2} M(T)$ and the $\geq$ ' becomes '=' when $h(t)$ is a constant.

See Appendix D for the proof.

\section{Sensitivity analysis}

Here we consider that component 1 has Weibull-distributed lifetime $F(t)=$ $1-\exp \left(-\lambda t^{b}\right), t>0$. The deterioration of component 2 is described by a homogeneous Gamma process which has been extensively applied in describing the system deterioration on the account of system erosion, corrosion, crack growth, etc. $([35,26,9])$. Assume that the density function is

$$
g_{\alpha t, \beta}(u)=\frac{\beta^{\alpha t} u^{\alpha t-1} e^{-\beta u}}{\Gamma(\alpha t)}, u \geq 0
$$

where

$$
\Gamma(\alpha)=\int_{0}^{\infty} u^{\alpha-1} e^{-u} d u
$$

Hence as it is mentioned in [29], the component 2 lifetime distribution $G_{\sigma_{L}}(t)$ is

$$
G_{\sigma_{L}}(t)=\frac{\Gamma(\alpha t, L \beta)}{\Gamma(\alpha t)}, t \geq 0
$$

where

$$
\Gamma(\alpha, x)=\int_{x}^{\infty} z^{\alpha-1} e^{-z} d z
$$

Let $\alpha=2, \beta=2, \lambda=\frac{1}{64}, b=2, L=8, c_{1}=1, c_{2}=5$. Tables 1 and 2 show the manufacturer's expected warranty costs within the warranty cycle under different warranty policies with respect to the failure interaction factor $\bar{r}$ and the initial warranty length $W$ respectively.

\begin{tabular}{|c|c|c|c|c|c|c|}
\hline $\bar{r}$ & 0 & 0.1 & 0.2 & 0.3 & 0.4 & 0.5 \\
\hline $\mathbb{E}(C(W))$ & 0.60 & 0.75 & 0.88 & 1.02 & 1.15 & 1.28 \\
\hline $\mathbb{E}\left(C^{R}(W)\right)$ & 0.62 & 0.78 & 0.95 & 1.13 & 1.33 & 1.53 \\
\hline
\end{tabular}

Table 1: The warranty costs of the manufacture under the non-renewing FRW and RFRW with respect to different values of $\bar{r}$ when $W=5$

We can observe that the expected warranty costs increase continuously with the failure interaction factor $(\bar{r})$ and the initial warranty length $(W)$ 


\begin{tabular}{|c|c|c|c|c|c|}
\hline$W$ & 4 & 5 & 6 & 7 & 8 \\
\hline $\mathbb{E}(C(W))$ & 0.58 & 1.02 & 1.70 & 2.65 & 3.72 \\
\cline { 2 - 6 } $\mathbb{E}\left(C^{R}(W)\right)$ & 0.61 & 1.13 & 2.14 & 4.13 & 8.09 \\
\hline
\end{tabular}

Table 2: The warranty costs of the manufacture under the non-renewing FRW and RFRW with respect to different values of $W$ when $\bar{r}=0.3$

respectively. It is more sensitive in regards of the RFRW policy, for example, when $\bar{r}=0.5$, the warranty cost under the RFRW is $(1.53-0.62) / 0.62=1.48$ fold larger than the cost of the system without failure interaction $(\bar{r}=0)$. For the non-renewing FRW case, this figure stands at $(1.28-0.60) / 0.60=1.13$. It is easy to understand that as $\bar{r}$ (or $W$ ) increases, more system major failure occurs within the warranty cycle which results in a rise of the warranty cost. Besides, the non-renewing FRW is always more economic to the manufacturer than the RFRW. The reason is due to that under the non-renewing FRW, the manufacturer pays in full of the warranty costs during a fixed time period $W$, whereas under the RFRW, a longer warranty cost coverage period is required.

It should deserve mentioning that the warranty cost during the warranty cycle could be served as a reference for the warranty budget. It is also important to consider the consumer' behaviour (for example his or her expected usage time period of the product) and the manufacturer's market share (for example the number of the consumers who prefers to come back for the product maintenance after the warranty expiration) for a general estimation of the warranty cost.

Suppose that the product is put into use for a time period $T$. The consumer comes back to the manufacturer (or the retailer, the seller, here we held the same regard between them) for the product maintenance after the expiration of the warranty cycle. He or she is charged $c_{11}, c_{22}$ respectively for the component 1 maintenance and the system replacement. Let $W=5, T=$ $10, \bar{r}=0.3, \alpha=2, \beta=2, \lambda=\frac{1}{64}, b=1, L=8, c_{1}=1, c_{2}=5, c_{11}=4, c_{22}=12$ be the basic parameter settings. The following figures and tables demonstrate the sensibilities of the manufacturer profits and the consumer costs by varying different system indicators and remaining others unchanged.

The following points can be highlighted.

- Figures 2 and 3 illustrate the warranty profits and costs for the manufacturer and the consumer with different values of $W$ and $\bar{r}$ respectively. Both the manufacturer profits and the consumer costs decrease with 


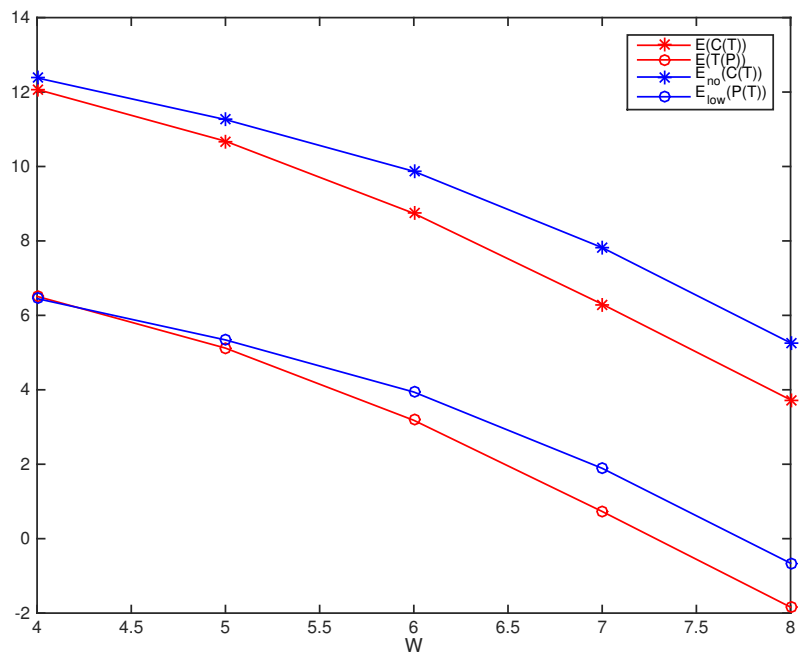

Figure 2: The manufacturer's warranty profits $\mathbb{E}(P(T))$ and $\mathbb{E}_{\text {low }}(P(T))$ and the consumer's warranty costs $\mathbb{E}(C(T))$ and $\mathbb{E}_{n o}(C(T))$ with different values of $W$

the initial warranty length $W$ and the failure interaction between components $\bar{r}$ respectively. It is easy to understand that as the the warranty period increases, the manufacturer has to cover more warranty costs. Consequently the expected manufacturer profit or the expected cost of the consumer decrease as the warranty period grows. The larger is $\bar{r}$, the shorter is the expected system lifetime. Therefore more system replacements are required during the warranty period $W$ and the product service time $T$ reducing the manufacturer warranty profit and the consumer's cost. Under each warranty policy, the difference between the consumer cost and the manufacturer profit is independent of $W$. The reason is that the difference between the consumer's cost and the manufacturer's profit is actually the manufacturer's maintenance cost during the product service time $T$ which is independent of $W$. The failure interaction between components has negative effect to the manufacturer profit. It is recommended to the engineers to eliminate the failure interaction between components.

- Table 3 describes the variations of the manufacturer profits and the consumer costs with respect to $(\lambda, b)$ the parameters of the Weibull distribution and the product service time $T$. For fixed $T$, under both 


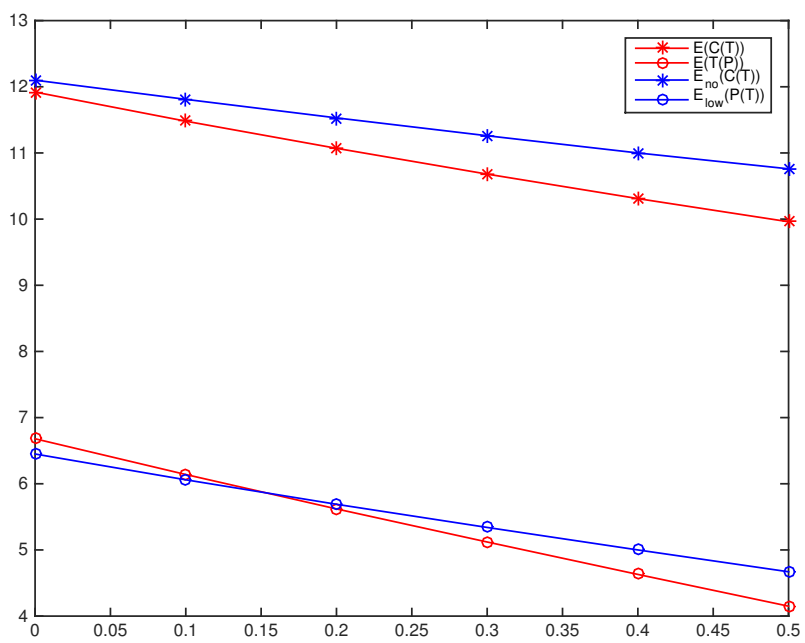

Figure 3: the manufacturer's warranty profits $\mathbb{E}(P(T))$ and $\mathbb{E}_{\text {low }}(P(T))$ and the consumer's warranty costs $\mathbb{E}(C(T))$ and $\mathbb{E}_{n o}(C(T))$ with different values of $\bar{r}$

the non-renewing FRW and RFRW, the warranty costs of the consumer are decreasing functions of $\lambda$ and $b$. It shows that given the product service time $T$, the consumer will prefer better quality and reliability of component 1 (small values of $\lambda$ and $b$ ) . Besides, from the perspective of the manufacturer, under the non-renewing FRW, for short product service time $(T=10)$, the manufacturer benefits less from a good quality component 1: the manufacturer's profit is 4.41 when the expected life time of component 1 is $\left.12\left(\lambda=\frac{1}{12}, b=1\right)\right)$ and the profit is more than 4.69 when the expected lifetime of component 1 is 6.93 $\left.\left(\lambda=\frac{1}{12}, b=2\right)\right)$. On the contrary, the corresponding manufacturer profits are -69.12 and 21.56 when the product service time is $T=50$. Therefore the service time of the product has a non negligable impact on the warranty profits which need to be well estimated in the devising of warranty strategies. It might be improper or even counterproductive to assume that the consumer uses the product for a long-run time horizon $(T=\infty)$.

- Table 4 represents the sensibility of the manufacturer profits and the consumer costs with different component 2 deterioration rates. The average deterioration rate is $\alpha / \beta$ in this case. It indicates that un- 


\begin{tabular}{|c|c|c|c|c|}
\hline$(\lambda, b, T)$ & $\mathbb{E}(C(T))$ & $\mathbb{E}(P(T))$ & $\mathbb{E}_{n o}(C(T))$ & $\mathbb{E}_{n o}(P(T))$ \\
\hline$\left(\frac{1}{64}, 2,10\right)$ & 10.68 & 5.12 & 11.26 & 5.34 \\
\hline$\left(\frac{1}{12}, 2,10\right)$ & 11.29 & 0.45 & 18.36 & 4.69 \\
\hline$\left(\frac{1}{12}, 1,10\right)$ & 9.55 & 4.16 & 9.84 & 4.41 \\
\hline$\left(\frac{1}{64}, 2,50\right)$ & 74.02 & 38.26 & 62.39 & 3.24 \\
\hline$\left(\frac{1}{12}, 2,50\right)$ & 82.67 & 20.56 & 123.79 & -69.12 \\
\hline$\left(\frac{1}{12}, 1,50\right)$ & 66.81 & 33.18 & 56.01 & 21.56 \\
\hline
\end{tabular}

Table 3: The warranty cost and profit under RFRW and the non-renewing FRW policies respectively with different values of $\lambda, b$ and $T$

\begin{tabular}{|c|c|c|c|c|}
\hline$(\alpha, \beta)$ & $\mathbb{E}(C(T))$ & $\mathbb{E}(P(T))$ & $\mathbb{E}_{n o}(C(T))$ & $\mathbb{E}_{n o}(P(T))$ \\
\hline$(2,1)$ & 4.98 & -4.81 & 11.20 & 1.40 \\
\hline$(1,1)$ & 7.65 & 3.65 & 7.82 & 3.81 \\
\hline
\end{tabular}

Table 4: The warranty cost and profit under RFRW and the non-renewing FRW policies respectively with different values of $\alpha$ and $\beta$.

der the non-renewing FRW, both the manufacturer and the consumer prefer component 2 with slow deterioration speed. However, it is more economic for the consumer owning a fast deteriorate speed when the RFRW is offered. Because in the situation that $\alpha=2, \beta=1$, $F s(W)=0.72$ which means with an opportunity of more than $70 \%$ the system is replaced by the manufacture with no charge and then the process repeats. However, this figure descends strikingly to 0.12 when $\alpha=1, \beta=1$. Besides, the system failure probability at $T$ is $F_{s}(T)=0.73$ which implies that the system major failure occurs frequently during the period $[W, T]$ during which the consumer has to take in charge the system replacement costs.

\section{Conclusions}

In this study, the warranty analysis of a two-component in series system is examined. Under both the non-renewing FRW and the RFRW, from the perspectives of the consumer and the manufacturer, the short-run total warranty profits and costs are calculated. The model is general enough to be applied to any 2-component in series system where the components have absolutely continuous lifetime distributions. A Numerical case is studied indicating that the failure interaction between components can affect the benefits of both the manufacturer and the consumer. The sensitivity analysis of other parameter 
settings are displayed. It provides a good reference to the decision-maker planning the warranty policy for the maximisation of the company profit.

\section{Appendix A. Proof of Theorem 3.1}

Proof. As component 1 failure occurs according to the non-homogeneous Poisson process with intensity rate $h(t)$, from the decomposition property of the non-homogeneous Poisson process, the number of component 1 failure inducing the minor system failure follows a non-homogeneous Poisson process with intensity rate $r h(t)$. Therefore before the major system failure, the expected failure number of component 1 by time $t$ can be given as $\mathbb{E}_{1}(t)=$ $r H(t)$. By conditioning on the first renewal time of the system $T_{1}$, we have

$$
\mathbb{E}\left(C(W) \mid T_{1}=t\right)= \begin{cases}c_{1} r H(W) & t>W \\ c_{2}+c_{1} r H(t)+\mathbb{E}(C(W-t)) & t \leq W\end{cases}
$$

where $\mathbb{E}(N(t))=\sum_{n=0}^{\infty} n p_{n}(t)$ is the expected maintenance cost of component 1 in $[0, t]$. Based on the law of total probability

$$
\begin{aligned}
\mathbb{E}(C(W)) & =c_{1} \bar{F}_{s}(W) r H(W) \\
& +\int_{0}^{W}\left\{c_{2}+c_{1} r H(t)+\mathbb{E}(C(W-t))\right\} \mathrm{d} F_{s}(t) \\
& =K(W)+\int_{0}^{W} \mathbb{E}(C(W-t)) \mathrm{d} F_{s}(t)
\end{aligned}
$$

where

$$
K(W)=c_{1} \bar{F}_{s}(W) r H(W)+\int_{0}^{W}\left(c_{2}+c_{1} r H(t)\right) \mathrm{d} F_{s}(t)
$$

From the renewal property, equation (A.1) is equal to

$$
\mathbb{E}(C(W))=K(W)+\int_{0}^{W} K(W-x) \mathrm{d} M(x)
$$

where $M(t)$ is the renewal function. The above equation is equal to

$$
\mathbb{E}(C(W))=\int_{0}^{W}[1+M(W-t)] k(t) \mathrm{d} t
$$

where $k(t)=c_{1} r h(t) \bar{F}_{s}(t)+c_{2} f_{s}(t)$. Therefore we obtained the expected warranty cost of the manufacturer under the non-renewing FRW.» 


\section{Appendix B. Proof of Theorem 3.2}

Proof. It can be noticed that $\mathbb{P}\left(N_{w}=n\right)=F_{s}^{n}(W) \bar{F}_{s}(W)$. Let $\mathbb{E}(C(t))$ be the expected warranty cost of the manufacturer during time period $t$ from the initial product sold moment. Then we have

$$
\begin{aligned}
& \mathbb{E}\left(C^{R}(W)\right)=\mathbb{E}\left[\mathbb{E}\left(C^{R}\left(W \mid N_{w}=n\right)\right)\right] \\
& =\sum_{n=0}^{\infty} F_{s}^{n}(W) \bar{F}_{s}(W) \mathbb{E}\left[C\left(\theta_{1}+\theta_{2}+\cdots+\theta_{n}+W\right)\right] \\
& =\sum_{n=0}^{\infty} F_{s}^{n}(W) \bar{F}_{s}(W) \int_{0}^{W} \cdots \int_{0}^{W}\left(n c_{2}+c_{1} r \sum_{i=1}^{n} H\left(t_{i}\right)+c_{1} r H(W)\right) \\
& \mathrm{d} F_{\theta}\left(t_{1}\right) \cdots \mathrm{d} F_{\theta}\left(t_{n}\right)=\sum_{n=0}^{\infty} \bar{F}_{s}(W) \\
& \left(n c_{2} F_{s}^{n}(W)+c_{1} r H(W) F_{s}^{n}(W)+n r c_{1} F_{s}^{n-1}(W) \int_{0}^{W} H(t) \mathrm{d} F_{s}(t)\right) \\
& =\sum_{n=0}^{\infty} \bar{F}_{s}(W) F_{s}^{n}(W)\left(n c_{2}+c_{1} r H(W)+n r c_{1} \frac{\int_{0}^{W} H(t) \mathrm{d} F_{s}(t)}{F_{s}(W)}\right)
\end{aligned}
$$

Therefore we obtain the expected warranty cost of the manufacturer under the RFRW. $\diamond$

\section{Appendix C. Proof of Theorem 3.3}

Proof. By conditioning on the first system renewal time $T_{1}$, we have

$$
\begin{aligned}
& \mathbb{E}\left(C(T) \mid T_{1}=t\right)= \\
& \left\{\begin{array}{c}
c_{11} r(H(T)-H(W)), t>T \\
c_{22}+c_{11} r(H(t)-H(W))+\mathbb{E}(C(T-t)), W<t \leq T \\
\mathbb{E}(C(T-t)), 0<t \leq W
\end{array}\right.
\end{aligned}
$$

By the law of total probability:

$$
\begin{aligned}
\mathbb{E}(C(T)) & =K(T)+\int_{0}^{T} K(T-u) \mathrm{d} M(u) \\
& =\int_{W}^{T}(1+M(T-u)) k(u) \mathrm{d} u
\end{aligned}
$$


where

$$
K(t)=c_{22}\left(F_{s}(t)-F_{s}(W)\right)+c_{11} \int_{W}^{t} \bar{F}_{s}(\theta) r h(\theta) \mathrm{d} \theta
$$

for $t \geq W$ and 0 otherwise. $k(u)$ is the derivative of $K(u)$ given as $k(u)=$ $\frac{\mathrm{d} K(u)}{\mathrm{d} u}=c_{22} f_{s}(u)+c_{11} \bar{F}_{s}(u) r h(u)$.

In addition, let be $\mathbb{E}\left(C_{0}(T)\right)$ the expected warranty cost caused by minor system failure by time $t$ for the manufacturer. By the similar method as in the calculation of $\mathbb{E}(C(T))$, we have

$$
\mathbb{E}\left(C_{0}(T)\right)=\int_{0}^{T}(1+M(T-t)) k_{1}(t) \mathrm{d} t
$$

where

$k_{1}(t)=\bar{F}_{s}(t) h(t), t>0$ and $M(t)$ is the renewal function related with $F_{s}(t)$. As the warranty profit of the manufacture is equivalent to the income gaining from the consumer's warranty payment minus the total warranty cost in $[0, T]$. So the manufacturer profit is deduced by substituting $\mathbb{E}(C(T))$, $\mathbb{E}\left(C_{0}(T)\right)$ and $c_{2} M(T)$ in the equation $\mathbb{E}(P(T))=\mathbb{E}(C(T))-\mathbb{E}\left(C_{0}(T)\right)-$ $c_{2} M(T) \diamond$

\section{Appendix D. Proof of Theorem 3.4}

Proof. Let $U,(U>W)$ be the time epoch when the system is firstly renewed after $W$ and $F_{U}(t)$ be its lifetime distribution function. Denote $\gamma(W)$ be the residual life to the system at time $W$, then $U=W+\gamma(W)$ and $F_{\gamma}(t)$ be its distribution function. Then

$$
F_{\gamma}(t)=F_{s}(W+t)-\int_{0}^{W} \bar{F}_{s}(W+t-x) \mathrm{d} M(x)
$$

and so

$$
F_{U}(t)=\left\{\begin{array}{lr}
0 & 0 \leq t \leq W \\
F_{s}(t)-\int_{0}^{W} \bar{F}_{s}(t-x) \mathrm{d} M(x) & W<t
\end{array}\right.
$$

where $M(W)=\sum_{n=1}^{\infty} F_{s}^{(n)}(W)$. By conditioning on the first consumer purchase time $U$ we have:

$$
\mathbb{E}_{n o}(C(T))=K_{0}(T)+\int_{W}^{T} K_{0}(T-x) \mathrm{d} M_{U}(x)
$$


where $K_{0}(t)=c_{22} F_{U}(t)+c_{11} \int_{W}^{t} \bar{F}_{U}(\theta) r h(\theta) \mathrm{d} \theta$ for $t>W$ and 0 otherwise, $M_{U}(t)$ is the renewal function related with $F_{U}(t)$.

Therefore the expected total cost of the ownership is derived by the law of total probability. The expected profit of the manufacturer is then obtained by as the revenue minus the maintenance cost by time $T$. It is easily seen that the system renewal cost during $T$ is $M(T)$. The minimal repair cost of component 1 is less or equal to $r H(T)$ when the component 1 lifetime distribution $F \in I F R$. For example, assume that the system is renewed once at $T_{1}$ during $T$. Let $T_{2}=T-T_{1}$, then the minimal repair cost is

$$
\begin{aligned}
r\left(H\left(T_{1}\right)+r H\left(T_{2}\right)\right) & =r\left(\int_{0}^{T_{1}} h(t) \mathrm{d} t+\int_{T_{1}}^{T} h\left(t-T_{1}\right) \mathrm{d} t\right) \\
& \leq r \int_{0}^{T} h(t) t=H(T)
\end{aligned}
$$

and ' $\geq$ ' becomes ' $=$ ' only when $h(t)$ is a constant. $\diamond$

[1] Bai J and Pham H (2006) Cost analysis on renewable full-service warranties for multi-component systems. European Journal of Operational Research 168(2): 492-508.

[2] Barros A, Bérenguer C and Grall A (2003) Optimization of replacement times using imperfect monitoring information. IEEE Transactions on Reliability 52(4): 523-533.

[3] Barros A, Berenguer C and Grall A (2006) A maintenance policy for two-unit parallel systems based on imperfect monitoring information. Reliability Engineering 85 System Safety 91(2): 131-136.

[4] Blischke WR and Murthy D (1992) Product warranty managementi: A taxonomy for warranty policies. European Journal of Operational Research 62(2): 127-148.

[5] Chien YH (2008) A general age-replacement model with minimal repair under renewing free-replacement warranty. European Journal of Operational Research 186(3): 1046-1058.

[6] Chien YH (2010) The effect of a pro-rata rebate warranty on the age replacement policy with salvage value consideration. IEEE Transactions on Reliability 59(2): 383-392. 
[7] Chien YH (2010) Optimal age for preventive replacement under a combined fully renewable free replacement with a pro-rata warranty. International Journal of Production Economics 124(1): 198-205.

[8] Darghouth M, Chelbi A and Ait-Kadi D (2012) A profit assessment model for equipment inspection and replacement under renewing free replacement warranty policy. International Journal of Production Economics 135(2): 899-906.

[9] Grall A, Bérenguer C and Dieulle L (2002) A condition-based maintenance policy for stochastically deteriorating systems. Reliability Engineering 86 System Safety 76(2): 167-180.

[10] Jain M and Maheshwari S (2006) Discounted costs for repairable units under hybrid warranty. Applied Mathematics and Computation 173(2): 887-901.

[11] Jhang JP and Sheu SH (2000) Optimal age and block replacement policies for a multi-component system with failure interaction. International Journal of Systems Science 31(5): 593-603.

[12] Jung KM, Park M and Park DH (2010) System maintenance cost dependent on life cycle under renewing warranty policy. Reliability Engineering \& System Safety 95(7): 816-821.

[13] Jung KM, Park M and Park DH (2012) Optimal maintenance strategy for non-renewing replacement-repair warranty. Applied Stochastic Models in Business and Industry 28(6): 607-614.

[14] Karim R and Suzuki K (2005) Analysis of warranty claim data: a literature review. International Journal of Quality $\&$ Reliability Management 22(7): 667-686.

[15] Lai MT and Chen YC (2008) Optimal replacement period of a two-unit system with failure rate interaction and external shocks. International Journal of Systems Science 39(1): 71-79.

[16] Li H, Deloux E and Dieulle L (2016) A condition-based maintenance policy for multi-component systems with lévy copulas dependence. Reliability Engineering \&5 System Safety 149: 44-55. 
[17] Liu B, Wu J and Xie M (2015) Cost analysis for multi-component system with failure interaction under renewing free-replacement warranty. European Journal of Operational Research 243(3): 874-882.

[18] Maaroufi G, Chelbi A and Rezg N (2012) A selective maintenance policy for multi-component systems with stochastic and economic dependence. In: 9th international conference of modelling, optimization and simulation.

[19] Murthy D and Blischke W (1992) Product warranty managementii: An integrated framework for study. European journal of operational research 62(3): 261-281.

[20] Murthy D and Blischke W (1992) Product warranty managementiii: a review of mathematical models. European Journal of Operational Research 63(1): 1-34.

[21] Murthy D and Djamaludin I (2002) New product warranty: A literature review. International Journal of Production Economics 79(3): 231-260.

[22] Murthy D and Nguyen D (1985) Study of two-component system with failure interaction. Naval Research Logistics Quarterly 32(2): 239-247.

[23] Nakagawa T and Murthy D (1993) Optimal replacement policies for a two-unit system with failure interactions. Revue française d'automatique, d'informatique et de recherche opérationnelle. Recherche opérationnelle 27(4): 427-438.

[24] Park M and Pham H (2010) Warranty cost analyses using quasi-renewal processes for multicomponent systems. IEEE Transactions on Systems, Man, and Cybernetics-Part A: Systems and Humans 40(6): 1329-1340.

[25] Park M and Pham H (2016) Cost models for age replacement policies and block replacement policies under warranty. Applied Mathematical Modelling 40(9): 5689-5702.

[26] Ponchet A, Fouladirad M and Grall A (2011) Maintenance policy on a finite time span for a gradually deteriorating system with imperfect improvements. Proceedings of the Institution of Mechanical Engineers, Part O: Journal of Risk and Reliability 225(2): 105-116. 
[27] Shafiee M and Chukova S (2013) Maintenance models in warranty: A literature review. European Journal of Operational Research 229(3): $561-572$.

[28] Shi H and Zeng J (2016) Real-time prediction of remaining useful life and preventive opportunistic maintenance strategy for multi-component systems considering stochastic dependence. Computers $\&$ Industrial Engineering 93: 192-204.

[29] Van Noortwijk J (2009) A survey of the application of gamma processes in maintenance. Reliability Engineering \& System Safety 94(1): 2-21.

[30] Wang GJ and Zhang YL (2009) A geometric process repair model for a two-component system with shock damage interaction. International Journal of Systems Science 40(11): 1207-1215.

[31] Wu CC, Chou CY and Huang C (2007) Optimal burn-in time and warranty length under fully renewing combination free replacement and pro-rata warranty. Reliability Engineering 8 System Safety 92(7): 914920.

[32] Wu S and Longhurst P (2011) Optimising age-replacement and extended non-renewing warranty policies in lifecycle costing. International Journal of Production Economics 130(2): 262-267.

[33] Yeh RH, Chen GC and Chen MY (2005) Optimal age-replacement policy for nonrepairable products under renewing free-replacement warranty. IEEE transactions on reliability 54(1): 92-97.

[34] Zequeira RI and Bérenguer C (2005) On the inspection policy of a twocomponent parallel system with failure interaction. Reliability Engineering 85 System Safety 88(1): 99-107.

[35] Zhu W, Fouladirad M and Bérenguer C (2015) Condition-based maintenance policies for a combined wear and shock deterioration model with covariates. Computers \&5 Industrial Engineering 85: 268-283. 